\title{
NEUROBIOLOGY OF AGING
}

\section{TABLE OF CONTENTS}

\begin{tabular}{lll}
\hline & & \\
- & Description & p.1 \\
$-\quad$ Audience & p.1 \\
- Impact Factor & p.1 \\
- Abstracting and Indexing & p.2 \\
- & Editorial Board & p.2 \\
- Guide for Authors & p.4
\end{tabular}

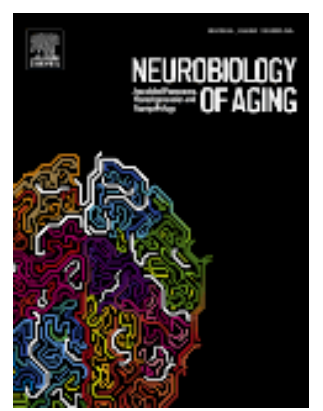

ISSN: $0197-4580$

\section{DESCRIPTION}

Neurobiology of Aging publishes the results of studies in behavior, biochemistry, cell biology, endocrinology, molecular biology, morphology, neurology, neuropathology, pharmacology, physiology and protein chemistry in which the primary emphasis involves mechanisms of nervous system changes with age or diseases associated with age. Reviews and primary research articles are included, occasionally accompanied by open peer commentary. Letters to the Editor and brief communications are also acceptable. Brief reports of highly time-sensitive material are usually treated as rapid communications in which case editorial review is completed within six weeks and publication scheduled for the next available issue. The accepted abbreviation for Neurobiology of Aging for bibliographic citation is Neurobiol. Aging

\section{Benefits to authors}

We also provide many author benefits, such as free PDFs, a liberal copyright policy, special discounts on Elsevier publications and much more. Please click here for more information on our author services.

Please see our Guide for Authors for information on article submission. If you require any further information or help, please visit our support pages: https://service.elsevier.com

Authors are also welcome to submit their manuscripts to the journal?s open access companion title, Aging Brain.

\section{AUDIENCE}

Neuroscientists, Molecular Biologists, Gerontologists.

\section{IMPACT FACTOR}

2021: 5.133 @ Clarivate Analytics Journal Citation Reports 2022 


\author{
Current Contents - Life Sciences \\ Elsevier BIOBASE \\ Science Citation Index \\ Embase \\ BIOSIS Citation Index \\ Research Alert \\ PsycINFO \\ PubMed/Medline \\ Web of Science \\ Scopus
}

\title{
EDITORIAL BOARD
}

\section{Editor-in-Chief}

David J. Madden, Duke University Medical Center, School of Medicine, Department of Psychiatry and Behavioral Sciences, Durham, North Carolina, United States of America

\section{Editor-in-Chief Emeritus}

Peter R. Rapp, National Institutes of Health (NIH), National Institute on Aging, Baltimore, Maryland, United States of America

\section{Managing Editor}

Pamela L. Yeh, Dartmouth College Geisel School of Medicine, Hanover, New Hampshire, United States of America

\section{Senior Editors}

\section{Alzheimer's Disease \& Other Dementias}

Ralph Nixon, Center for Dementia Research, Orangeburg, New York, United States of America

Akihiko Takashima, Gakushuin University, Faculty of Science, Department of Life Sciences Laboratory for Alzheimer's Disease, Toshima, Japan

\section{Behavior, Cognition \& Neurophysiology}

Michelle M. Adams, Bilkent University, Ankara, Turkey

\section{Genetic Reports}

Mark R. Cookson, National Institutes of Health, Bethesda, Maryland, United States of America

\section{Imaging}

Giovanni B. Frisoni, HUG and UniGeneva Intern Med-Psychiatry, Geneve, Switzerland

David J. Madden, Duke University Medical Center, School of Medicine, Department of Psychiatry and Behavioral Sciences, Durham, North Carolina, United States of America

\section{Stress Signaling \& Neuroplasticity}

Steven Barger, University of Arkansas for Medical Sciences, Department of Neurobiology and Developmental Sciences, Little Rock, Arkansas, United States of America

\section{Structure}

Patrick R. Hof, Icahn School of Medicine at Mount Sinai Fishberg, Department of Neuroscience, New York, New York, United States of America

\section{Translational Preclinical and Clinical Research}

Raymond T. Bartus, RTBioconsultants, Inc., San Diego, California, United States of America

\section{Biomarkers of Neurocognitive Disorders}

Giovanni Frisoni, Geneva, Switzerland

\section{Reviewing Editors}

Ilana Bennett, University of California Riverside, Riverside, California, United States of America

Paul D. Coleman, Arizona State University, Tempe, Arizona, United States of America

Joshua Goh, National Taiwan University College of Medicine, Taipei, Taiwan

Alan A. Hartley, Scripps College, Claremont, California, United States of America

Jonas Persson, Aging Research Center, Stockholm, Sweden

Michela Pievani, IRCCS S John of God Fatebenefratelli Centre, Brescia, Italy 
Julia Spaniol, Toronto Metropolitan University, Department of Psychology, Toronto, Ontario, Canada

\section{Editorial Advisory Board}

Gene Alexander, Tucson, Arizona, United States of America

Jesus Avila, Madrid, Spain

Heiko Braak, Ulm, Germany

Ashley I. Bush, Parkville, Victoria, Australia

Gemma Casadesus Smith, Kent, Ohio, United States of America

Colin Combs, Grand Forks, North Dakota, United States of America

Carl Cotman, Irvine, California, United States of America

Charles DeCarli, Sacramento, California, United States of America

Dennis Dickson, Jacksonville, Florida, United States of America

Jian-Dong Ding, Shanghai, China

Jianqing Ding, Shanghai, China

James Eberwine, Philadelphia, Pennsylvania, United States of America

Caleb Finch, Los Angeles, California, United States of America

Dorothy Flood, West Chester, Pennsylvania, United States of America

Pierluigi Gambetti, Cleveland, Ohio, United States of America

Ezio Giacobini, Genève, Switzerland

Gunner K. Gouras, Lund, Sweden

Karl Herrup, Cleveland, Ohio, New Jersey, United States of America

Bradley Hyman, Boston, Massachusetts, United States of America

Donald Ingram, Baton Rouge, Louisiana, United States of America

Khalid Iqbal, Staten Island, New York, United States of America

Terry Jernigan, La Jolla, California, United States of America

Zaven Khachaturian, Bethesda, Maryland, United States of America

Frank LaFerla, Irvine, California, United States of America

Philip Landfield, Lexington, Kentucky, United States of America

John H. Morrison, Davis, California, New York, United States of America

Elliott Mufson, Phoenix, Arizona, Illinois, United States of America

Giulio Pasinetti, New York, New York, United States of America

Daniel Peterson, North Chicago, Illinois, United States of America

Huntington Potter, Aurora, Colorado, United States of America

Remi Quirion, Montréal, Quebec, Canada

Joseph Rogers, Phoenix, Arizona, United States of America

Paul Sanberg, Tampa, Florida, United States of America

Robert Sapolsky, Stanford, California, United States of America

Michael Shelanski, New York, New York, United States of America

Yong Shen, Qingdao, China

J. Randall Slemmon, Titusville, New Jersey, New York, United States of America

David Smith, Oxford, United Kingdom

Michael Sofroniew, Los Angeles, California, United States of America

Alexis M. Stranahan, Augusta, Georgia, United States of America

Dick Swaab, Amsterdam, Netherlands

Rudolph Tanzi, Boston, Massachusetts, United States of America

B.J. Traynor, Bethesda, Maryland, United States of America 


\section{GUIDE FOR AUTHORS}

\section{AIMS AND SCOPE}

Neurobiology of Aging publishes the results of studies in behavior, biochemistry, cell biology, endocrinology, molecular biology, morphology, neurology, neuropathology, pharmacology, physiology and protein chemistry in which the primary emphasis involves mechanisms of nervous system changes with age or diseases associated with age. Only original articles will be accepted. Reviews and primary research articles are included, occasionally accompanied by open peer commentary. Letters to the Editor and brief communications are also acceptable. Brief reports of highly time-sensitive material are usually treated as rapid communications in which case editorial review is completed within 6 weeks and publication scheduled for the next available issue. Negative Results will be published as one journal page (3 double-spaced typed manuscript pages), with supplementary material to be posted at the journal website.

Authors are referred to the following published editorial policy statements: Coleman, P.D. How old is old? Neurobiol. Aging 25:1;2004. Coleman, P.D.; Finch, C.E.; Joseph, J. The need for multiple time points in aging studies. Neurobiol. Aging 25:3-4;2004. Finch, C.E. Middle-age: An evolving frontier in gerontology. Neurobiol. Aging 12:1-2;1991. West, M.J. New stereological methods for counting neurons. Neurobiol. Aging 14:275-285;1993. West, M.J.; Coleman, P.D. How to count. Neurobiol. Aging 17:503;1996. Frisoni, G.B.; Coleman, P.D. Mild cognitive impairment: instructions for use at Neurobiology of Aging. Neurobiol. Aging 32:761-762;2011.

\section{GUIDELINES}

Genetic Analysis of Disease in the Era of Whole Genome Analysis and Public Databases. Over the past 5 years genetic analysis has changed almost beyond recognition. We now have the technology to assess association between any phenotype and alleles across the genome in a single analysis. Furthermore, these data are stored in publicly available databases such as dbGAP (www.ncbi.nlm.nih.gov/gap) and Alzgene (www.alzgene.org) where they are accessible and can be used in ongoing meta-analyses. In this environment, researchers should consider carefully the extent to which analyses they report substantively contribute to the literature.

In the future, we will expect authors of any manuscripts submitted to access these databases before submission. While there are circumstances when limited analyses are appropriate, in general, clearly whole genome analyses are the way forward and there is no doubt that findings which come out of such studies are more reliable than those which come from candidate gene analyses. Additionally, we caution against the overinterpretation of analyses of secondary phenotypes (such as age of onset, or rate of cognitive decline).

In studies where whole genome analyses are reported, we will always expect full summary statistics to be made available alongside the publication.

We note that for many major phenotypes, there remain no whole genome reports. Clear examples include Alzheimer'??s disease in populations outside of Europeans. We would welcome such studies.

Genetic Reports. It is our wish to provide rapid review of high quality-genetic studies for traits and conditions related to normal and diseased aging brain, whether these are positive or negative in outcome.

Genetic analysis and technologies have moved on and we want the studies we publish to be definitive. With this in mind, we suggest the following should be considered when you are submitting to Neurobiology of Aging:

(1) Does the study assess the whole gene? We would suggest that any analysis should include a haplotypic analysis of the whole gene of interest rather than single SNPs unless the SNP tested is believed to be the functional SNP.

(2) How is your study powered? This question should be addressed whether the study is positive or negative. In general, for dichotomous traits one should aim at reasonable numbers (cases and controls each of 500 is a good rule of thumb). These numbers can usually be achieved through collaboration. 
(3) Is your study a hypothesis-generating or a hypothesis-testing study? Does it inform as to mechanism? In general, reviewers and editors are very wary of effects that purport to be present in only a subset of cases. Such contrasts should be pre-specified and designs appropriately powered to test for the effect of sex and other variables. A clear negative study has value. Digging around in data to generate positive findings does the field a disservice.

(4) Have your sample series been used in other studies? Clearly these should always be referenced so the audience can assess how much risk may have been reported to have been found in any sample series.

(5) Are there online data sources in which you can also assess your SNPs? There are now online resources of case control series for Alzheimer's disease, Parkinson's disease and brain gene expression. The number of these resources is increasing all the time: any association studies for which there is already data should reference and include these data, perhaps as secondary sample series.

These are not rules, but guidelines.

NB: The full text of Genetic Reports manuscripts submitted after November 30th 2010, if accepted, will be published as e-pub only. The full text of such manuscripts will appear online within 40-50 days of acceptance, with the abstract appearing in the next available print edition as well. The abstract in print will contain appropriate reference to the complete e-pub manuscript.

Biomarker Reports. As a journal devoted to aging and neurobiology, Neurobiology of Aging uses certain criteria for evaluating priority for publishing work on biomarkers. These include more than one of the following criteria:

(1) Novelty of the biomarker and relationship to disease mechanisms.

(2) The potential of the marker (based on evidence in the literature or in the manuscript) for directly revealing insight into disease mechanisms.

(3) The clinical potential of the marker for differential diagnosis or prediction of disease progression (based on data in the manuscript).

(4) The reliability of the supporting data based on size of the sample studied and statistical validation.

\section{Submission checklist}

You can use this list to carry out a final check of your submission before you send it to the journal for review. Please check the relevant section in this Guide for Authors for more details.

\section{Ensure that the following items are present:}

One author has been designated as the corresponding author with contact details:

- E-mail address

- Full postal address

All necessary files have been uploaded:

Manuscript:

- Include keywords

- All figures (include relevant captions)

- All tables (including titles, description, footnotes)

- Ensure all figure and table citations in the text match the files provided

- Indicate clearly if color should be used for any figures in print

Graphical Abstracts / Highlights files (where applicable)

Supplemental files (where applicable)

Further considerations

- Manuscript has been 'spell checked' and 'grammar checked'

- All references mentioned in the Reference List are cited in the text, and vice versa

- Permission has been obtained for use of copyrighted material from other sources (including the Internet)

- A competing interests statement is provided, even if the authors have no competing interests to declare

- Journal policies detailed in this guide have been reviewed

- Referee suggestions and contact details provided, based on journal requirements 
For further information, visit our Support Center.

\section{BEFORE YOU BEGIN}

\section{Ethics in publishing}

Please see our information on Ethics in publishing.

\section{Declaration of interest}

All authors must disclose any financial and personal relationships with other people or organizations that could inappropriately influence (bias) their work. Examples of potential competing interests include employment, consultancies, stock ownership, honoraria, paid expert testimony, patent applications/registrations, and grants or other funding. Authors must disclose any interests in two places: 1. A summary declaration of interest statement in the title page file (if double anonymized) or the manuscript file (if single anonymized). If there are no interests to declare then please state this: 'Declarations of interest: none'. 2. Detailed disclosures as part of a separate Declaration of Interest form, which forms part of the journal's official records. It is important for potential interests to be declared in both places and that the information matches. More information.

\section{Declaration of generative AI in scientific writing}

The below guidance only refers to the writing process, and not to the use of AI tools to analyse and draw insights from data as part of the research process.

Where authors use generative artificial intelligence (AI) and AI-assisted technologies in the writing process, authors should only use these technologies to improve readability and language. Applying the technology should be done with human oversight and control, and authors should carefully review and edit the result, as AI can generate authoritative-sounding output that can be incorrect, incomplete or biased. AI and AI-assisted technologies should not be listed as an author or co-author, or be cited as an author. Authorship implies responsibilities and tasks that can only be attributed to and performed by humans, as outlined in Elsevier's AI policy for authors.

Authors should disclose in their manuscript the use of AI and AI-assisted technologies in the writing process by following the instructions below. A statement will appear in the published work. Please note that authors are ultimately responsible and accountable for the contents of the work.

\section{Disclosure instructions}

Authors must disclose the use of generative AI and AI-assisted technologies in the writing process by adding a statement at the end of their manuscript in the core manuscript file, before the References list. The statement should be placed in a new section entitled 'Declaration of Generative AI and AIassisted technologies in the writing process'.

Statement: During the preparation of this work the author(s) used [NAME TOOL / SERVICE] in order to [REASON]. After using this tool/service, the author(s) reviewed and edited the content as needed and take(s) full responsibility for the content of the publication.

This declaration does not apply to the use of basic tools for checking grammar, spelling, references etc. If there is nothing to disclose, there is no need to add a statement.

\section{Submission declaration and verification}

Submission of an article implies that the work described has not been published previously (except in the form of an abstract, a published lecture or academic thesis, see 'Multiple, redundant or concurrent publication' for more information), that it is not under consideration for publication elsewhere, that its publication is approved by all authors and tacitly or explicitly by the responsible authorities where the work was carried out, and that, if accepted, it will not be published elsewhere in the same form, in English or in any other language, including electronically without the written consent of the copyrightholder. To verify originality, Elsevier may check your article using the originality detection service Crossref Similarity Check and share the results with the Editor-in-Chief. Please note, you do not have to upload this file with your submission."

\section{Preprints}

Please note that preprints can be shared anywhere at any time, in line with Elsevier's sharing policy. Sharing your preprints e.g. on a preprint server will not count as prior publication (see 'Multiple, redundant or concurrent publication' for more information). 


\section{Preprint posting on SSRN}

In support of Open Science, this journal offers its authors a free preprint posting service. Preprints provide early registration and dissemination of your research, which facilitates early citations and collaboration.

During submission to Editorial Manager, you can choose to release your manuscript publicly as a preprint on the preprint server SSRN once it enters peer-review with the journal. Your choice will have no effect on the editorial process or outcome with the journal. Please note that the corresponding author is expected to seek approval from all co-authors before agreeing to release the manuscript publicly on SSRN.

You will be notified via email when your preprint is posted online and a Digital Object Identifier (DOI) is assigned. Your preprint will remain globally available free to read whether the journal accepts or rejects your manuscript.

For more information about posting to SSRN, please consult the SSRN Terms of Use and FAQs.

\section{Use of inclusive language}

Inclusive language acknowledges diversity, conveys respect to all people, is sensitive to differences, and promotes equal opportunities. Content should make no assumptions about the beliefs or commitments of any reader; contain nothing which might imply that one individual is superior to another on the grounds of age, gender, race, ethnicity, culture, sexual orientation, disability or health condition; and use inclusive language throughout. Authors should ensure that writing is free from bias, stereotypes, slang, reference to dominant culture and/or cultural assumptions. We advise to seek gender neutrality by using plural nouns ("clinicians, patients/clients") as default/wherever possible to avoid using "he, she," or "he/she." We recommend avoiding the use of descriptors that refer to personal attributes such as age, gender, race, ethnicity, culture, sexual orientation, disability or health condition unless they are relevant and valid. When coding terminology is used, we recommend to avoid offensive or exclusionary terms such as "master", "slave", "blacklist" and "whitelist". We suggest using alternatives that are more appropriate and (self-) explanatory such as "primary", "secondary", "blocklist" and "allowlist". These guidelines are meant as a point of reference to help identify appropriate language but are by no means exhaustive or definitive.

\section{Reporting sex- and gender-based analyses}

\section{Reporting guidance}

For research involving or pertaining to humans, animals or eukaryotic cells, investigators should integrate sex and gender-based analyses (SGBA) into their research design according to funder/ sponsor requirements and best practices within a field. Authors should address the sex and/or gender dimensions of their research in their article. In cases where they cannot, they should discuss this as a limitation to their research's generalizability. Importantly, authors should explicitly state what definitions of sex and/or gender they are applying to enhance the precision, rigor and reproducibility of their research and to avoid ambiguity or conflation of terms and the constructs to which they refer (see Definitions section below). Authors can refer to the Sex and Gender Equity in Research (SAGER) guidelines and the SAGER guidelines checklist. These offer systematic approaches to the use and editorial review of sex and gender information in study design, data analysis, outcome reporting and research interpretation - however, please note there is no single, universally agreed-upon set of guidelines for defining sex and gender.

\section{Definitions}

Sex generally refers to a set of biological attributes that are associated with physical and physiological features (e.g., chromosomal genotype, hormonal levels, internal and external anatomy). A binary sex categorization (male/female) is usually designated at birth ("sex assigned at birth"), most often based solely on the visible external anatomy of a newborn. Gender generally refers to socially constructed roles, behaviors, and identities of women, men and gender-diverse people that occur in a historical and cultural context and may vary across societies and over time. Gender influences how people view themselves and each other, how they behave and interact and how power is distributed in society. Sex and gender are often incorrectly portrayed as binary (female/male or woman/man) and unchanging whereas these constructs actually exist along a spectrum and include additional sex categorizations and gender identities such as people who are intersex/have differences of sex development (DSD) or identify as non-binary. Moreover, the terms "sex" and "gender" can be ambiguous-thus it is important 
for authors to define the manner in which they are used. In addition to this definition guidance and the SAGER guidelines, the resources on this page offer further insight around sex and gender in research studies.

\section{Author contributions}

For transparency, we encourage authors to submit an author statement file outlining their individual contributions to the paper using the relevant CRediT roles: Conceptualization; Data curation; Formal analysis; Funding acquisition; Investigation; Methodology; Project administration; Resources; Software; Supervision; Validation; Visualization; Roles/Writing - original draft; Writing - review \& editing. Authorship statements should be formatted with the names of authors first and CRediT role(s) following. More details and an example.

\section{Changes to authorship}

Authors are expected to consider carefully the list and order of authors before submitting their manuscript and provide the definitive list of authors at the time of the original submission. Any addition, deletion or rearrangement of author names in the authorship list should be made only before the manuscript has been accepted and only if approved by the journal Editor. To request such a change, the Editor must receive the following from the corresponding author: (a) the reason for the change in author list and (b) written confirmation (e-mail, letter) from all authors that they agree with the addition, removal or rearrangement. In the case of addition or removal of authors, this includes confirmation from the author being added or removed.

Only in exceptional circumstances will the Editor consider the addition, deletion or rearrangement of authors after the manuscript has been accepted. While the Editor considers the request, publication of the manuscript will be suspended. If the manuscript has already been published in an online issue, any requests approved by the Editor will result in a corrigendum.

\section{Article transfer service}

This journal uses the Elsevier Article Transfer Service to find the best home for your manuscript. This means that if an editor feels your manuscript is more suitable for an alternative journal, you might be asked to consider transferring the manuscript to such a journal. The recommendation might be provided by a Journal Editor, a dedicated Scientific Managing Editor, a tool assisted recommendation, or a combination. If you agree, your manuscript will be transferred, though you will have the opportunity to make changes to the manuscript before the submission is complete. Please note that your manuscript will be independently reviewed by the new journal. More information.

\section{Copyright}

Upon acceptance of an article, authors will be asked to complete a 'Journal Publishing Agreement' (see more information on this). An e-mail will be sent to the corresponding author confirming receipt of the manuscript together with a 'Journal Publishing Agreement' form or a link to the online version of this agreement.

Subscribers may reproduce tables of contents or prepare lists of articles including abstracts for internal circulation within their institutions. Permission of the Publisher is required for resale or distribution outside the institution and for all other derivative works, including compilations and translations. If excerpts from other copyrighted works are included, the author(s) must obtain written permission from the copyright owners and credit the source(s) in the article. Elsevier has preprinted forms for use by authors in these cases.

For gold open access articles: Upon acceptance of an article, authors will be asked to complete a 'License Agreement' (more information). Permitted third party reuse of gold open access articles is determined by the author's choice of user license.

\section{Author rights}

As an author you (or your employer or institution) have certain rights to reuse your work. More information.

Elsevier supports responsible sharing

Find out how you can share your research published in Elsevier journals. 


\section{Role of the funding source}

You are requested to identify who provided financial support for the conduct of the research and/or preparation of the article and to briefly describe the role of the sponsor(s), if any, in study design; in the collection, analysis and interpretation of data; in the writing of the report; and in the decision to submit the article for publication. If the funding source(s) had no such involvement, it is recommended to state this.

\section{Open access}

Please visit our Open Access page for more information.

\section{Elsevier Researcher Academy}

Researcher Academy is a free e-learning platform designed to support early and mid-career researchers throughout their research journey. The "Learn" environment at Researcher Academy offers several interactive modules, webinars, downloadable guides and resources to guide you through the process of writing for research and going through peer review. Feel free to use these free resources to improve your submission and navigate the publication process with ease.

\section{Language (usage and editing services)}

Please write your text in good English (American or British usage is accepted, but not a mixture of these). Authors who feel their English language manuscript may require editing to eliminate possible grammatical or spelling errors and to conform to correct scientific English may wish to use the English Language Editing service available from Elsevier's Author Services.

\section{Submission}

Our online submission system guides you stepwise through the process of entering your article details and uploading your files. The system converts your article files to a single PDF file used in the peer-review process. Editable files (e.g., Word, LaTeX) are required to typeset your article for final publication. All correspondence, including notification of the Editor's decision and requests for revision, is sent by e-mail.

\section{Submit your article}

Please submit your article via https://www.editorialmanager.com/nba/default.aspx.

\section{Suggesting reviewers}

Please submit the names and institutional e-mail addresses of several potential reviewers.

You should not suggest reviewers who are colleagues, or who have co-authored or collaborated with you during the last three years. Editors do not invite reviewers who have potential competing interests with the authors. Further, in order to provide a broad and balanced assessment of the work, and ensure scientific rigor, please suggest diverse candidate reviewers who are located in different countries/ regions from the author group. Also consider other diversity attributes e.g. gender, race and ethnicity, career stage, etc. Finally, you should not include existing members of the journal's editorial team, of whom the journal are already aware.

Note: the editor decides whether or not to invite your suggested reviewers.

\section{PREPARATION}

Use of word processing software

It is important that the file be saved in the native format of the word processor used. The text should be in single-column format. Keep the layout of the text as simple as possible. Most formatting codes will be removed and replaced on processing the article. In particular, do not use the word processor's options to justify text or to hyphenate words. However, do use bold face, italics, subscripts, superscripts etc. When preparing tables, if you are using a table grid, use only one grid for each individual table and not a grid for each row. If no grid is used, use tabs, not spaces, to align columns. The electronic text should be prepared in a way very similar to that of conventional manuscripts (see also the Guide to Publishing with Elsevier). Note that source files of figures, tables and text graphics will be required whether or not you embed your figures in the text. See also the section on Electronic artwork.

To avoid unnecessary errors you are strongly advised to use the 'spell-check' and 'grammar-check' functions of your word processor. 


\section{Negative results}

Negative results will be published as one journal page ( 3 double-spaced typed manuscript pages), with supplementary material to be posted at the journal website. The 3 double-spaced pages should include a brief abstract, a brief introduction, a few sentences of methods, core data and discussion of the data. If room allows, an abbreviated reference list with the most critical references should also be included in these 3 double-spaced pages.

Supplementary material for the website should include amore detailed introduction, more details of the methods, the complete reference list and any additional data. The supplementary material should be sufficient to convince the interested reader of the validity and reliability of the results. It should be made clear which material is for printing in thejournal and which is supplementary material for the website. Since the net effect of a Negative Result is to discourage repetition, the standards for acceptance as a Negative Result will be highly demanding (see Announcement "Negative results can be valuable", Neurobiol. Aging 25(10):iii;2004).

\section{Length of paper}

The Editors insist upon clear, concise statements of facts and conclusions. Regular manuscripts should be no longer that 10 printed journal pages (30 doubled-spaced pages, including references, figures and tables) and should include only the most essential figures and tables. Brief Communications should be restricted to eight double-spaced pages (including references, figures and tables) and should not present more than one figure and one table, or two figures, or two tables. Fragmentation of material into numerous short reports is discouraged.

\section{Subdivision - numbered sections}

Divide your article into clearly defined and numbered sections. Subsections should be numbered 1.1 (then $1.1 .1,1.1 .2, \ldots), 1.2$, etc. (the abstract is not included in section numbering). Use this numbering also for internal cross-referencing: do not just refer to 'the text'. Any subsection may be given a brief heading. Each heading should appear on its own separate line.

\section{Introduction}

State the objectives of the work and provide an adequate background, avoiding a detailed literature survey or a summary of the results.

\section{Material and methods}

Provide sufficient details to allow the work to be reproduced by an independent researcher. Methods that are already published should be summarized, and indicated by a reference. If quoting directly from a previously published method, use quotation marks and also cite the source. Any modifications to existing methods should also be described.

\section{Theory/calculation}

A Theory section should extend, not repeat, the background to the article already dealt with in the Introduction and lay the foundation for further work. In contrast, a Calculation section represents a practical development from a theoretical basis.

\section{Results}

Results should be clear and concise.

\section{Discussion}

This should explore the significance of the results of the work, not repeat them. A combined Results and Discussion section is often appropriate. Avoid extensive citations and discussion of published literature.

\section{Conclusions}

The main conclusions of the study may be presented in a short Conclusions section, which may stand alone or form a subsection of a Discussion or Results and Discussion section.

\section{Appendices}

If there is more than one appendix, they should be identified as $A, B$, etc. Formulae and equations in appendices should be given separate numbering: Eq. (A.1), Eq. (A.2), etc.; in a subsequent appendix, Eq. (B.1) and so on. Similarly for tables and figures: Table A.1; Fig. A.1, etc.

\section{Essential title page information}

- Title. Concise and informative. Titles are often used in information-retrieval systems. Avoid abbreviations and formulae where possible. 
- Author names and affiliations. Please clearly indicate the given name(s) and family name(s) of each author and check that all names are accurately spelled. You can add your name between parentheses in your own script behind the English transliteration. Present the authors' affiliation addresses (where the actual work was done) below the names. Indicate all affiliations with a lowercase superscript letter immediately after the author's name and in front of the appropriate address. Provide the full postal address of each affiliation, including the country name and, if available, the e-mail address of each author.

- Corresponding author. Clearly indicate who will handle correspondence at all stages of refereeing and publication, also post-publication. This responsibility includes answering any future queries about Methodology and Materials. Ensure that the e-mail address is given and that contact details are kept up to date by the corresponding author.

- Present/permanent address. If an author has moved since the work described in the article was done, or was visiting at the time, a 'Present address' (or 'Permanent address') may be indicated as a footnote to that author's name. The address at which the author actually did the work must be retained as the main, affiliation address. Superscript Arabic numerals are used for such footnotes.

\section{Highlights}

Highlights are mandatory for this journal as they help increase the discoverability of your article via search engines. They consist of a short collection of bullet points that capture the novel results of your research as well as new methods that were used during the study (if any). Please have a look at the examples here: example Highlights.

Highlights should be submitted in a separate editable file in the online submission system. Please use 'Highlights' in the file name and include 3 to 5 bullet points (maximum 85 characters, including spaces, per bullet point).

\section{Abstract}

A concise and factual abstract is required. The abstract, not to exceed 170 words, should state briefly the purpose of the research, the principal results and major conclusions. An abstract is often presented separately from the article, so it must be able to stand alone. For this reason, References should be avoided, but if essential, then cite the author(s) and year(s). Also, non-standard or uncommon abbreviations should be avoided, but if essential they must be defined at their first mention in the abstract itself.

\section{Graphical abstract}

Although a graphical abstract is optional, its use is encouraged as it draws more attention to the online article. The graphical abstract should summarize the contents of the article in a concise, pictorial form designed to capture the attention of a wide readership. Graphical abstracts should be submitted as a separate file in the online submission system. Image size: Please provide an image with a minimum of $531 \times 1328$ pixels $(h \times w)$ or proportionally more. The image should be readable at a size of $5 \times$ $13 \mathrm{~cm}$ using a regular screen resolution of $96 \mathrm{dpi}$. Preferred file types: TIFF, EPS, PDF or MS Office files. You can view Example Graphical Abstracts on our information site.

\section{Keywords}

Immediately after the abstract, provide a maximum of 6 keywords, using American spelling and avoiding general and plural terms and multiple concepts (avoid, for example, 'and', 'of'). Be sparing with abbreviations: only abbreviations firmly established in the field may be eligible. These keywords will be used for indexing purposes.

\section{Abbreviations}

Define abbreviations that are not standard in this field in a footnote to be placed on the first page of the article. Such abbreviations that are unavoidable in the abstract must be defined at their first mention there, as well as in the footnote. Ensure consistency of abbreviations throughout the article.

\section{Acknowledgements}

Collate acknowledgements in a separate section at the end of the article before the references and do not, therefore, include them on the title page, as a footnote to the title or otherwise. List here those individuals who provided help during the research (e.g., providing language help, writing assistance or proof reading the article, etc.).

\section{Formatting of funding sources}

List funding sources in this standard way to facilitate compliance to funder's requirements: 
Funding: This work was supported by the National Institutes of Health [grant numbers xxxx, yyyy]; the Bill \& Melinda Gates Foundation, Seattle, WA [grant number zzzz]; and the United States Institutes of Peace [grant number aaaa].

It is not necessary to include detailed descriptions on the program or type of grants and awards. When funding is from a block grant or other resources available to a university, college, or other research institution, submit the name of the institute or organization that provided the funding.

If no funding has been provided for the research, it is recommended to include the following sentence:

This research did not receive any specific grant from funding agencies in the public, commercial, or not-for-profit sectors.

Units

Follow internationally accepted rules and conventions: use the international system of units (SI). If other units are mentioned, please give their equivalent in SI.

Math formulae

Please submit math equations as editable text and not as images. Present simple formulae in line with normal text where possible and use the solidus $(/)$ instead of a horizontal line for small fractional terms, e.g., X/Y. In principle, variables are to be presented in italics. Powers of e are often more conveniently denoted by exp. Number consecutively any equations that have to be displayed separately from the text (if referred to explicitly in the text).

\section{Footnotes}

Footnotes should be used sparingly. Number them consecutively throughout the article. Many word processors can build footnotes into the text, and this feature may be used. Otherwise, please indicate the position of footnotes in the text and list the footnotes themselves separately at the end of the article. Do not include footnotes in the Reference list.

\section{Artwork}

Electronic artwork

General points

- Make sure you use uniform lettering and sizing of your original artwork.

- Embed the used fonts if the application provides that option.

- Aim to use the following fonts in your illustrations: Arial, Courier, Times New Roman, Symbol, or use fonts that look similar.

- Number the illustrations according to their sequence in the text.

- Use a logical naming convention for your artwork files.

- Provide captions to illustrations separately.

- Size the illustrations close to the desired dimensions of the published version.

- Submit each illustration as a separate file.

- Ensure that color images are accessible to all, including those with impaired color vision.

A detailed guide on electronic artwork is available.

You are urged to visit this site; some excerpts from the detailed information are given here. Formats

If your electronic artwork is created in a Microsoft Office application (Word, PowerPoint, Excel) then please supply 'as is' in the native document format.

Regardless of the application used other than Microsoft Office, when your electronic artwork is finalized, please 'Save as' or convert the images to one of the following formats (note the resolution requirements for line drawings, halftones, and line/halftone combinations given below):

EPS (or PDF): Vector drawings, embed all used fonts.

TIFF (or JPEG): Color or grayscale photographs (halftones), keep to a minimum of $300 \mathrm{dpi}$.

TIFF (or JPEG): Bitmapped (pure black \& white pixels) line drawings, keep to a minimum of $1000 \mathrm{dpi}$. TIFF (or JPEG): Combinations bitmapped line/half-tone (color or grayscale), keep to a minimum of 500 dpi.

\section{Please do not:}

- Supply files that are optimized for screen use (e.g., GIF, BMP, PICT, WPG); these typically have a low number of pixels and limited set of colors;

- Supply files that are too low in resolution;

- Submit graphics that are disproportionately large for the content. 


\section{Color artwork}

Please make sure that artwork files are in an acceptable format (TIFF (or JPEG), EPS (or PDF), or MS Office files) and with the correct resolution. If, together with your accepted article, you submit usable color figures then Elsevier will ensure, at no additional charge, that these figures will appear in color online (e.g., ScienceDirect and other sites) regardless of whether or not these illustrations are reproduced in color in the printed version. For color reproduction in print, you will receive information regarding the costs from Elsevier after receipt of your accepted article. Please indicate your preference for color: in print or online only. Further information on the preparation of electronic artwork.

Figure captions

Ensure that each illustration has a caption. Supply captions separately, not attached to the figure. A caption should comprise a brief title (not on the figure itself) and a description of the illustration. Keep text in the illustrations themselves to a minimum but explain all symbols and abbreviations used.

\section{Tables}

Please submit tables as editable text and not as images. Tables can be placed either next to the relevant text in the article, or on separate page(s) at the end. Number tables consecutively in accordance with their appearance in the text and place any table notes below the table body. Be sparing in the use of tables and ensure that the data presented in them do not duplicate results described elsewhere in the article. Please avoid using vertical rules and shading in table cells.

\section{References}

Citation in text

Please ensure that every reference cited in the text is also present in the reference list (and vice versa). Any references cited in the abstract must be given in full. Unpublished results and personal communications are not recommended in the reference list, but may be mentioned in the text. If these references are included in the reference list they should follow the standard reference style of the journal and should include a substitution of the publication date with either 'Unpublished results' or 'Personal communication'. Citation of a reference as 'in press' implies that the item has been accepted for publication.

\section{Reference links}

Increased discoverability of research and high quality peer review are ensured by online links to the sources cited. In order to allow us to create links to abstracting and indexing services, such as Scopus, Crossref and PubMed, please ensure that data provided in the references are correct. Please note that incorrect surnames, journal/book titles, publication year and pagination may prevent link creation. When copying references, please be careful as they may already contain errors. Use of the DOI is highly encouraged.

A DOI is guaranteed never to change, so you can use it as a permanent link to any electronic article. An example of a citation using DOI for an article not yet in an issue is: VanDecar J.C., Russo R.M., James D.E., Ambeh W.B., Franke M. (2003). Aseismic continuation of the Lesser Antilles slab beneath northeastern Venezuela. Journal of Geophysical Research, https://doi.org/10.1029/2001JB000884. Please note the format of such citations should be in the same style as all other references in the paper.

\section{Web references}

As a minimum, the full URL should be given and the date when the reference was last accessed. Any further information, if known (DOI, author names, dates, reference to a source publication, etc.), should also be given. Web references can be listed separately (e.g., after the reference list) under a different heading if desired, or can be included in the reference list.

\section{Data references}

This journal encourages you to cite underlying or relevant datasets in your manuscript by citing them in your text and including a data reference in your Reference List. Data references should include the following elements: author name(s), dataset title, data repository, version (where available), year, and global persistent identifier. Add [dataset] immediately before the reference so we can properly identify it as a data reference. The [dataset] identifier will not appear in your published article. 


\section{Preprint references}

Where a preprint has subsequently become available as a peer-reviewed publication, the formal publication should be used as the reference. If there are preprints that are central to your work or that cover crucial developments in the topic, but are not yet formally published, these may be referenced. Preprints should be clearly marked as such, for example by including the word preprint, or the name of the preprint server, as part of the reference. The preprint DOI should also be provided.

\section{References in a special issue}

Please ensure that the words 'this issue' are added to any references in the list (and any citations in the text) to other articles in the same Special Issue.

\section{Reference management software}

Most Elsevier journals have their reference template available in many of the most popular reference management software products. These include all products that support Citation Style Language styles, such as Mendeley and Zotero, as well as EndNote. Using the word processor plug-ins from these products, authors only need to select the appropriate journal template when preparing their article, after which citations and bibliographies will be automatically formatted in the journal's style. If no template is yet available for this journal, please follow the format of the sample references and citations as shown in this Guide.

\section{Reference style}

Text: All citations in the text should refer to:

1. Single author: the author's name (without initials, unless there is ambiguity) and the year of publication;

2. Two authors: both authors' names and the year of publication;

3. Three or more authors: first author's name followed by 'et al.' and the year of publication.

Citations may be made directly (or parenthetically). Groups of references should be listed first alphabetically, then chronologically.

Examples: 'as demonstrated in wheat (Allan, 2000a, 2000b, 1999; Allan and Jones, 1999). Kramer et al. (2010) have recently shown ....'

List: References should be arranged first alphabetically and then further sorted chronologically if necessary. More than one reference from the same author(s) in the same year must be identified by the letters 'a', ' $b$ ', 'c', etc., placed after the year of publication.

Examples:

Reference to a journal publication:

Van der Geer J, Hanraads JAJ, Lupton RA. The art of writing a scientific article. J Sci Commun 2010;163:51-9.

Reference to a book:

Strunk Jr W, White EB. The elements of style. 4th ed. New York: Longman; 2000.

Reference to a chapter in an edited book:

Mettam GR, Adams LB. How to prepare an electronic version of your article. In: Jones BS, Smith RZ, editors. Introduction to the electronic age. New York: E-Publishing Inc; 2009. p. 281-304.

Note shortened form for last page number. e.g., 51-9. Do not use et al the names of all authors must be included. For further details you are referred to "Uniform Requirements for Manuscripts submitted to Biomedical Journals" (J Am Med Assoc 1997;277:927-34) (see also http://www.nlm.nih.gov/bsd/uniform_requirements.html).

Reference to dataset:

Oguro, M, Imahiro, S, Saito, S, Nakashizuka, T. Mortality data for Japanese oak wilt disease and surrounding forest compositions, Mendeley Data, v1; 2015.

Journal abbreviations source

Journal names should be abbreviated according to the List of Title Word Abbreviations.

Video

Elsevier accepts video material and animation sequences to support and enhance your scientific research. Authors who have video or animation files that they wish to submit with their article are strongly encouraged to include links to these within the body of the article. This can be done in the same way as a figure or table by referring to the video or animation content and noting in the body text where it should be placed. All submitted files should be properly labeled so that they directly relate to the video file's content. In order to ensure that your video or animation material is directly usable, please provide the file in one of our recommended file formats with a preferred maximum size of 150 MB per file, 1 GB in total. Video and animation files supplied will be published online in the electronic version of your article in Elsevier Web products, including ScienceDirect. Please supply 
'stills' with your files: you can choose any frame from the video or animation or make a separate image. These will be used instead of standard icons and will personalize the link to your video data. For more detailed instructions please visit our video instruction pages. Note: since video and animation cannot be embedded in the print version of the journal, please provide text for both the electronic and the print version for the portions of the article that refer to this content.

\section{Data visualization}

Include interactive data visualizations in your publication and let your readers interact and engage more closely with your research. Follow the instructions here to find out about available data visualization options and how to include them with your article.

\section{Supplementary material}

Supplementary material such as applications, images and sound clips, can be published with your article to enhance it. Submitted supplementary items are published exactly as they are received (Excel or PowerPoint files will appear as such online). Please submit your material together with the article and supply a concise, descriptive caption for each supplementary file. If you wish to make changes to supplementary material during any stage of the process, please make sure to provide an updated file. Do not annotate any corrections on a previous version. Please switch off the 'Track Changes' option in Microsoft Office files as these will appear in the published version.

\section{Research data}

This journal encourages and enables you to share data that supports your research publication where appropriate, and enables you to interlink the data with your published articles. Research data refers to the results of observations or experimentation that validate research findings. To facilitate reproducibility and data reuse, this journal also encourages you to share your software, code, models, algorithms, protocols, methods and other useful materials related to the project.

Below are a number of ways in which you can associate data with your article or make a statement about the availability of your data when submitting your manuscript. If you are sharing data in one of these ways, you are encouraged to cite the data in your manuscript and reference list. Please refer to the "References" section for more information about data citation. For more information on depositing, sharing and using research data and other relevant research materials, visit the research data page.

\section{Data linking}

If you have made your research data available in a data repository, you can link your article directly to the dataset. Elsevier collaborates with a number of repositories to link articles on ScienceDirect with relevant repositories, giving readers access to underlying data that gives them a better understanding of the research described.

There are different ways to link your datasets to your article. When available, you can directly link your dataset to your article by providing the relevant information in the submission system. For more information, visit the database linking page.

For supported data repositories a repository banner will automatically appear next to your published article on ScienceDirect.

In addition, you can link to relevant data or entities through identifiers within the text of your manuscript, using the following format: Database: xxxx (e.g., TAIR: AT1G01020; CCDC: 734053; PDB: 1XFN).

\section{Data statement}

To foster transparency, we encourage you to state the availability of your data in your submission. This may be a requirement of your funding body or institution. If your data is unavailable to access or unsuitable to post, you will have the opportunity to indicate why during the submission process, for example by stating that the research data is confidential. The statement will appear with your published article on ScienceDirect. For more information, visit the Data Statement page.

\section{AFTER ACCEPTANCE}

\section{Online proof correction}

To ensure a fast publication process of the article, we kindly ask authors to provide us with their proof corrections within two days. Corresponding authors will receive an e-mail with a link to our online proofing system, allowing annotation and correction of proofs online. The environment is similar to 
MS Word: in addition to editing text, you can also comment on figures/tables and answer questions from the Copy Editor. Web-based proofing provides a faster and less error-prone process by allowing you to directly type your corrections, eliminating the potential introduction of errors.

If preferred, you can still choose to annotate and upload your edits on the PDF version. All instructions for proofing will be given in the e-mail we send to authors, including alternative methods to the online version and PDF.

We will do everything possible to get your article published quickly and accurately. Please use this proof only for checking the typesetting, editing, completeness and correctness of the text, tables and figures. Significant changes to the article as accepted for publication will only be considered at this stage with permission from the Editor. It is important to ensure that all corrections are sent back to us in one communication. Please check carefully before replying, as inclusion of any subsequent corrections cannot be guaranteed. Proofreading is solely your responsibility.

\section{Offprints}

The corresponding author will, at no cost, receive 25 free paper offprints, or alternatively a customized Share Link providing 50 days free access to the final published version of the article on ScienceDirect. The Share Link can be used for sharing the article via any communication channel, including email and social media. For an extra charge, paper offprints can be ordered via the offprint order form which is sent once the article is accepted for publication. Corresponding authors who have published their article gold open access do not receive a Share Link as their final published version of the article is available open access on ScienceDirect and can be shared through the article DOI link.

\section{AUTHOR INQUIRIES}

Visit the Elsevier Support Center to find the answers you need. Here you will find everything from Frequently Asked Questions to ways to get in touch.

You can also check the status of your submitted article or find out when your accepted article will be published. 